\title{
Identification of Two Cold Water-Soluble Polysaccharides from the Stems of Ephedra sinica Stapf
}

\author{
Yonggang Xia, Jun Liang, Bingyou Yang, Qiuhong Wang, Haixue Kuang* \\ Key Laboratory of Chinese Materia Medica, Heilongjiang University of Chinese Medicine, \\ Ministry of Education, Harbin, China \\ Email: hxkuang@hotmail.com \\ Received December 4, 2010; revised December 8, 2010; accepted December 10, 2010
}

\begin{abstract}
Two polysaccharides (ESP-A1 and ESP-A2) were isolated from the cold water extract of Ephedra sinica Stapf and purified through ethanol precipitation, deproteinization and by ion exchange and gel-filtration chromatography. Their molecular weight was determined using high performance size exclusion chromatography and evaporative light scattering detector (HPSEC-ELSD) and their monosaccharide composition was analyzed by high performance capillary electrophoresis (HPCE) based on pre-column derivatization with 1phenyl-3-methyl-5-pyrazolone (PMP). It was shown that ESP-A1 consisted of xylose, arabinose, glucose, mannose and galactose and ESP-A2 consisted of xylose, arabinose, rhamnose and galactose, in a molar ratio (\%) of 3.2: 61.1: 11.1: 12.9: 11.6 and 20.6: 67.7: 5.0: 6.7, respectively. The molecular weights (Mw) of ESP-A1 and ESP-A2 were $5.83 \times 10^{4} \mathrm{Da}$ and more than $200 \times 10^{4} \mathrm{Da}$, respectively. To the best of our knowledge, two neutral polysaccharides are now being reported for the first time in this study.
\end{abstract}

Keywords: Ephedra sinica Stapf, Polysaccharides, Isolation and Purification, Molecular Weight, Monosaccharide Composition

\section{Introduction}

Ephedrae herba is the dried stem of Ephedra sinica Stapf, E. intermedia Schrenk et C. A. Mey. and E. equisetina Bge., which belongs to the Ephedraceae family [1]. It is a crude drug widely used in Traditional Chinese Medicine (TCM) for thousands of years for its medicinal qualities as a diaphoretic, diuretic, anti-asthmatic to treat allergies, asthma, pneumonia, bronchitis, hay fever and colds $[2,3]$. It is well-known that a series of ephedrine alkaloids have for a long time been considered as the pharmacologically active ingredients of $E$. sinica for treatment of various diseases and symptoms [4], but they cannot account for all the effects mentioned above and the water-soluble polysaccharides are also demonstrated to be one of the main bioactive constituents of E. sinica. Polysaccharides from Ephedrae herba are a class of macromolecules that have been shown obvious immunosuppressive effects by carbon clearance test, delayed-type hypersensitivity reaction and serum hemolysin analysis in vivo [2,3]. In the present study, we extracted, isolated and purified the polysaccharide components from the stems of $E$. sinica and determined their molecular weight as well as their monosaccharide composition so as to further investigate its mechanism of action.

\section{Experimental}

\subsection{Materials and Reagents}

The dry stems of E. sinica were collected in March 2007 from Datong of Shanxi Province, China and identified by Prof. Zhenyue Wang of Heilongiiang University of Chinese Medicine. The voucher specimen (20070016) was deposited at Herbarium of Heilongjiang University of Chinese Medicine, Harbin, P. R. China.

D-mannose (Man), L-rhamnose (Rha), D-glucose (Glc), D-galactose (Gal), L-arabinose (Ara), D-xylose (Xyl), Dglucuronic acid (GlcUA), D-galacturonic acid (GalUA), sulfuric acid $\left(\mathrm{H}_{2} \mathrm{SO}_{4}\right)$, were purchased from Sigma (St. Louis, USA). 1-Phenyl-3-methyl-5-pyrazolone (PMP), purchased from Beijing Reagent Plant (Beijing, China), was recrystallized twice from chromatographic grade methanol before use. DEAE Sepharose Fast Flow, Sephacryl S-400 HR, and Sephacryl S-200 HR were from the Pharmacia Co. (Sweden). All other chemicals were of the highest grade available. 


\subsection{Isolation of Polysaccharides from E. sinica}

The dry stems of E. sinica were ground to powders, and submitted to sequential extractions as follows: dry powders $(1.0 \mathrm{~kg})$ were extracted 3 times with 10 vol of $95 \%$ EtOH under reflux for $3 \mathrm{~h}$ each time to remove lipids. The residue was dried in air and then extracted 3 times with 10 vol of distilled water for $24 \mathrm{~h}$ (each time) at $4^{\circ} \mathrm{C}$. The combined aqueous extracts were filtered, concentrated 10 -fold, and $95 \%$ EtOH added to final concentration of $80 \%$. The precipitate was dissolved in $600 \mathrm{~mL}$ of water and deproteinated 15 times with $200 \mathrm{~mL}$ of $5: 1$ chloroform- $n$-butanol as described by Staub [5]. The resulting aqueous fraction was extensively dialyzed (cut-off $M_{\mathrm{w}} 3500 \mathrm{Da}$ ) against tap water for $48 \mathrm{~h}$ and distilled water for $48 \mathrm{~h}$ and precipitated again by adding a 5 fold volume of ethanol. After centrifugation, the precipitate was washed with anhydrous ethanol and then dissolved in water and lyophilised to yield the crude polysaccharide A (8.5 g) was collected by centrifugation (3000 rpm, $10 \mathrm{~min}, 20^{\circ} \mathrm{C}$ ).

Crude polysaccharide A (3.0 g) was dissolved in distilled water and passed through two series connected resin columns (Amberlite $\mathrm{FPA} 90-\mathrm{Cl}\left(\mathrm{Cl}^{-}\right.$form) and $\mathrm{Am}-$ berlite IRC-84 $\left(\mathrm{H}^{+}\right.$form $)$) eluting with distilled water and $1.0 \mathrm{M} \mathrm{NaCl}$ to yield fractions Fr. A1 (1.2 g) and Fr. A2 (0.9 g), respectively. Fr. A1 (1.0 g) was chromatographed over DEAE-Sepharose F. F eluting with distilled water and $0.2 \mathrm{M} \mathrm{NaCl}$ to yield subfractions Fr. A1-1 (400.0 mg) and Fr. A1-2 (380.0 mg), respectively. Fr. A1-1 (400.0 mg) was further purified by gel-permeation chromatography on a high resolution Sephacryl S-400 eluting with distilled water to afford Fr.-A1-1-1 (160.0 $\mathrm{mg}$ ) and Fr.-A1-1-2 (180.0 mg). Fr.-A1-1-1 (160.0 mg) was further purified by DEAE-Sepharose F. F eluting with distilled water to afford ESP-A1 (130.0 mg). Fr. -A1-1-2 was further purified by Sephacryl S-200 eluting with distilled water to afford ESP-A2 (145.0 mg).

\subsection{Identification on Purity of Polysaccharides and Molecular Weight Determination}

The molecular mass of the polysaccharide $(5 \mathrm{mg} / \mathrm{mL})$ was determined by high performance liquid chromatography (HPLC), using Waters 2695 HPLC and Alltech ELSD 2000 detector. The separation was carried out on a Shodex sugar KS-805 column $(8.0 \mathrm{~mm} \times 300 \mathrm{~mm}, 17$ $\mu \mathrm{m})$ coupled with a Shodex KS-G guard column (6 mm $\times 50 \mathrm{~mm}, 7 \mu \mathrm{m})$. The Dextran standards (T-10, T-40, T-70, T-500, T-2000) were used for the calibration curve. The isocratic elution was employed using water with 0.5 $\mathrm{mL} / \mathrm{min}$ at $30^{\circ} \mathrm{C}$ and the injection volume was $10 \mu \mathrm{L}$. While the drift tube temperature for ELSD was set at 116
${ }^{\circ} \mathrm{C}$, the nitrogen flow rate was $3.3 \mathrm{~L} / \mathrm{min}$ for the determination of polysaccharides. Their purities were over $98 \%$ by HPLC analysis. Total carbohydrate contents in purified samples were determined by phenol-sulfuric acid colorimetric method using glucose as the standard (Dubois, Gilles, Hamilton, Rebers, \& Smith 1956). Proteins in the polysaccharides were detected by the method of UV absorption on a TU-1800PC spectrophotometer (Beijing Purkinje General Instrument Co., Ltd., China).

\subsection{Analysis of Monosaccharide Composition}

Monosaccharide composition was analyzed according to the following procedure: Each polysaccharide sample $\left(20 \mathrm{mg}\right.$ ) was dissolved in $2 \mathrm{ml}$ of $2.0 \mathrm{M} \mathrm{H}_{2} \mathrm{SO}_{4}$ in an ampoule $(5 \mathrm{ml})$. The ampoule was sealed under a nitrogen atmosphere and kept in $110^{\circ} \mathrm{C}$ to hydrolyze the polysaccharide into component monosaccharides for $6 \mathrm{~h}$, then cooled to room temperature and neutralized with 2 $\mathrm{ml}$ of 4.0 $\mathrm{M}$ sodium hydroxide. The reaction mixture was diluted to $5 \mathrm{ml}$ with deionized water and was centrifugalized at $1000 \mathrm{rpm}$ for $5 \mathrm{~min}$. Then the supernatant was ready for the following experiments.

PMP derivatization of monosaccharides was carried out as described previously [6]. $200 \mu \mathrm{l}$ of individual standard monosaccharide, or mix standard monosaccharide solutions, or the hydrolyzed polysaccharide samples were placed in the $2.0 \mathrm{~mL}$ centrifuge tubes, respectively, then $0.5 \mathrm{M}$ methanol solution $(100 \mu \mathrm{l})$ of PMP and $0.3 \mathrm{M}$ aqueous sodium hydroxide $(100 \mu \mathrm{l})$ were added to each. Each mixture was allowed to react for $30 \mathrm{~min}$ at $70^{\circ} \mathrm{C}$ water bath, then cooled to room temperature and neutralized with $100 \mu \mathrm{l}$ of $0.3 \mathrm{M} \mathrm{HCl}$. The resulting solution was performed on liquid-liquid extraction with same volume of isoamyl acetate (two times) and chloroform (one time), respectively. After being shaken vigorously and centrifuged, the organic phase was carefully discarded to remove the excess reagents. Then the aqueous layer was filtered through a $0.45 \mu \mathrm{m}$ membrane and diluted with water before HPCE analysis.

The analysis of PMP-labeled monosaccharides was carried out on a P/ACE MDQ capillary electrophoresis instrument (Beckman Coulter, Fullerton, CA, USA). An integrated P/ACE 32 Karat Station (software version 4.0) was used to perform the data collection and to control the operational variables of the system. Separation was carried out in an unmodified fused silica capillary $(48.5 \mathrm{~cm}$ $\times 50 \mu \mathrm{m}$ i.d., effective length $40 \mathrm{~cm}$ ) with direct UV monitoring using a photodiode array detector at wavelength $254 \mathrm{~nm}$ including $35 \mathrm{mM}$ borate at $\mathrm{pH}$ 10.02, capillary temperature $25^{\circ} \mathrm{C}$ and applied voltage $20 \mathrm{kV}$. The molar ratio of the component monosaccharides is calculated as follows. The correction factor is shown in the 
equation: $f_{i / n}=\left(m_{i} / A_{i}\right) /\left(m_{n} / A_{n}\right)$, where $A_{i}$ and $A_{n}$ are the values of their peak areas in the standard monosaccharide, respectively. $m_{i}$ and $m_{n}$ are the values of their weights of the standard monosaccharide, respectively. The molar ratio value is shown in the equation: $R_{i / n}=f_{i / n}$ $\times\left(A_{i}^{\prime} / A_{n}^{\prime}\right)$, where $A_{i}^{\prime} / A_{n}^{\prime}$ is the ratio value of peak area for the component monosaccharide of tested samples and $f_{i / n}$ is the correction factor.

\section{Results and Discussion}

\subsection{Isolation of Polysaccharides from the Stems of E. sinica}

Though there are numerous literature reports on the extraction of crude polysaccharides from TCMs, few reports have studied the isolation, purification, molecular weight and monosaccharide composition of neutral polysaccharides from the stems of $E$. sinica [3,4]. In the present work, the extraction and isolation of ephedrae polysaccharides was performed by cold water and ethanol precipitation to yield crude polysaccharide, then the Sevag method was used to remove protein components after re-dissolution of the crude polysaccharides. The solution was then dialyzed against against tap water for $48 \mathrm{~h}$ and distilled water for $48 \mathrm{~h}$ and precipitated by adding a 5 fold volume of ethanol. The precipitate was collected by centrifugation and washed successively with absolute ethanol and acetone to give a light yellow powder. For additional purification the ephedrae polysaccharides were subjected to two series connected resin columns (Amberlite FPA90-Cl ( $\mathrm{Cl}^{-}$form) and Amberlite IRC-84 $\left(\mathrm{H}^{+}\right.$form $)$) eluting with distilled water to yield fractions Fr. A1. Fr. A1 continues DEAE-Sepharose F. F column chromatography eluting with distilled water. Each $5 \mathrm{~mL}$ eluted fraction was collected and the content of sugar was monitored using the phenol-sulfuric acid method. Fractions 5-60 and 140-160 were combined and designated as the Fr. A1-1 and Fr. A1-2 fraction (Figure 1). ESP-A1 and ESP-A2 were acquired at last by EAESepharose F. F column and Sephacryl S-200 column, respectively. Figures $\mathbf{2}$ and $\mathbf{3}$ showed that one main peak was found in the elution trace by this method.

\subsection{Molecular Weight Determination of Polysaccharide}

The two polysaccharides appeared as only a single and symmetrical sharp peak in high performance gel permeation chromatography by HPLC-ELSD with Shodex sugar KS-805 column (Figure 4). The average molecular weights were ca.5.83 $\times 10^{4} \mathrm{Da}$ and $>200 \times 10^{4} \mathrm{Da}$ for ESP-A1 and ESP-A2, respectively, by reference to the

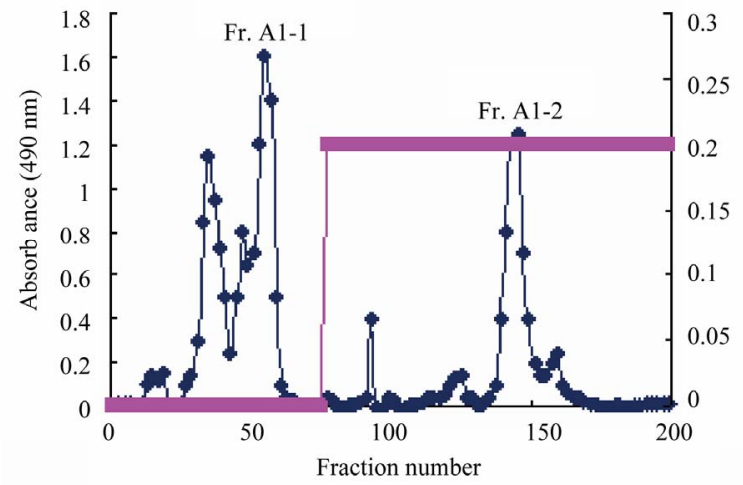

Figure 1. Elution profile of Fr. A on DEAE-Sepharose F. F column.

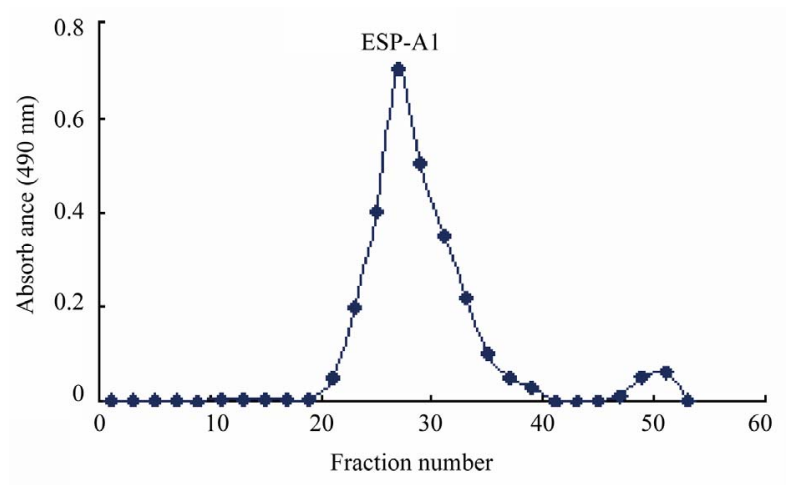

Figure 2. Elution profile of ESP-A1 from DEAE-Sepharose F. F column.

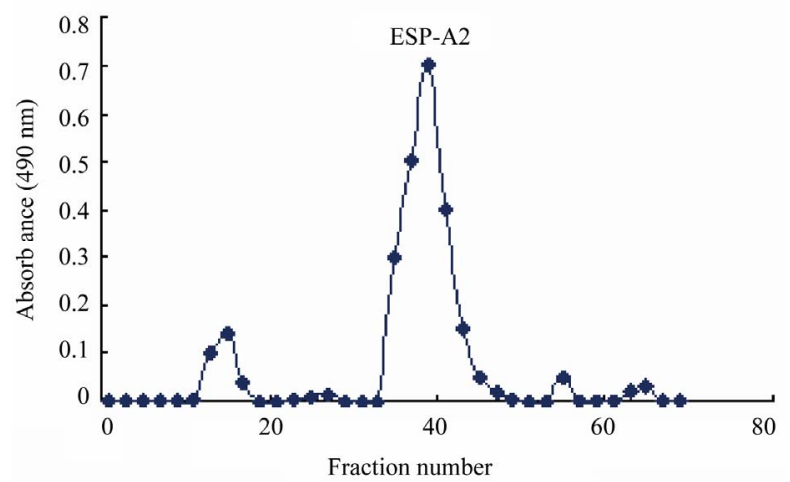

Figure 3. Elution profile of ESP-A2 from Sephacryl S-200 column.

calibration curve $\left(y=-2.2303, x+31.68, r^{2}=0.9936\right)$ made from a Dextran T-series standard of known molecular weight (T10, T40 T70, T500, T2000). These polysaccharides showed negative Fehling's reagent and iodine-potassium iodide reactions, indicating that they didn't contain reducing sugar and starch-type polysaccharide. All two polysaccharides had negative responses to the Bradford test and no absorption at $280 \mathrm{~nm}$ in the UV spectrum, indicating the absence of protein. 


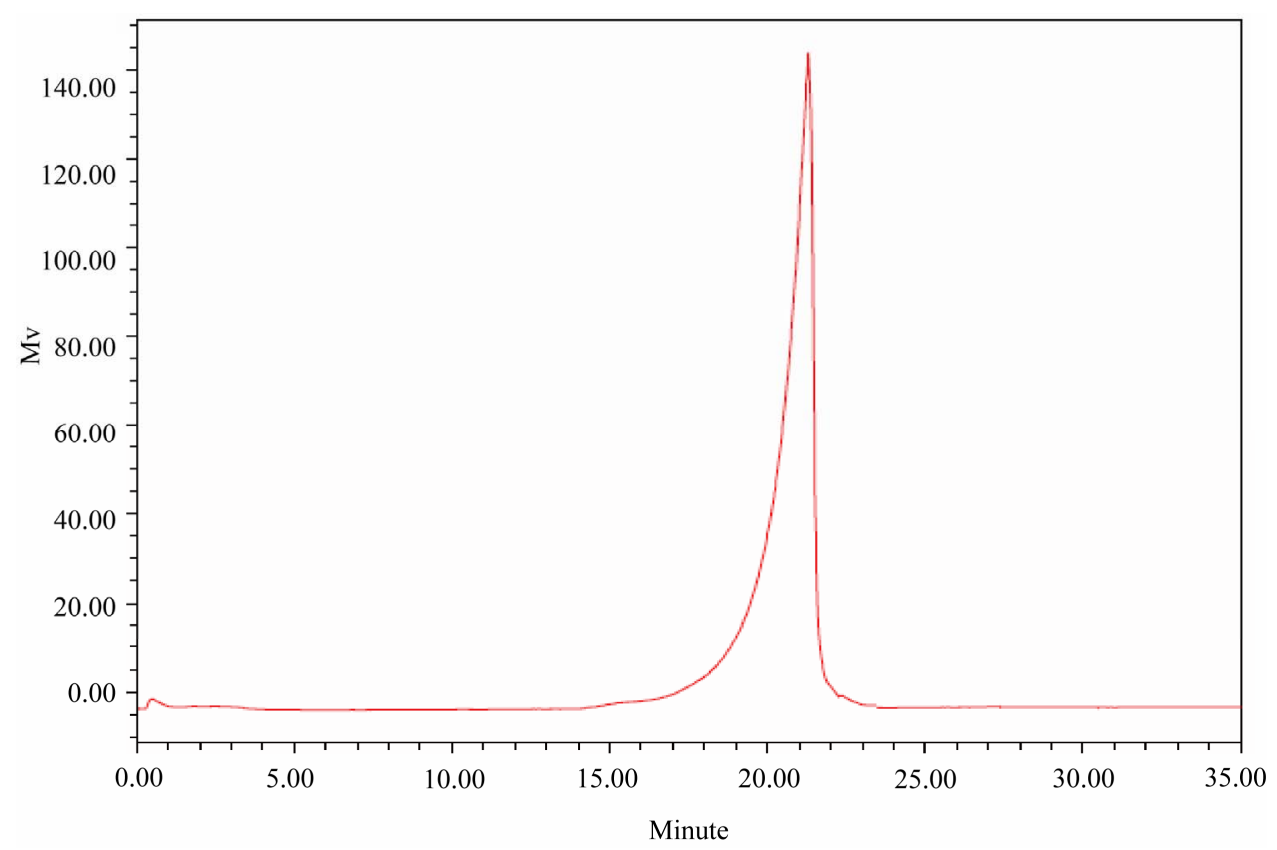

(a)

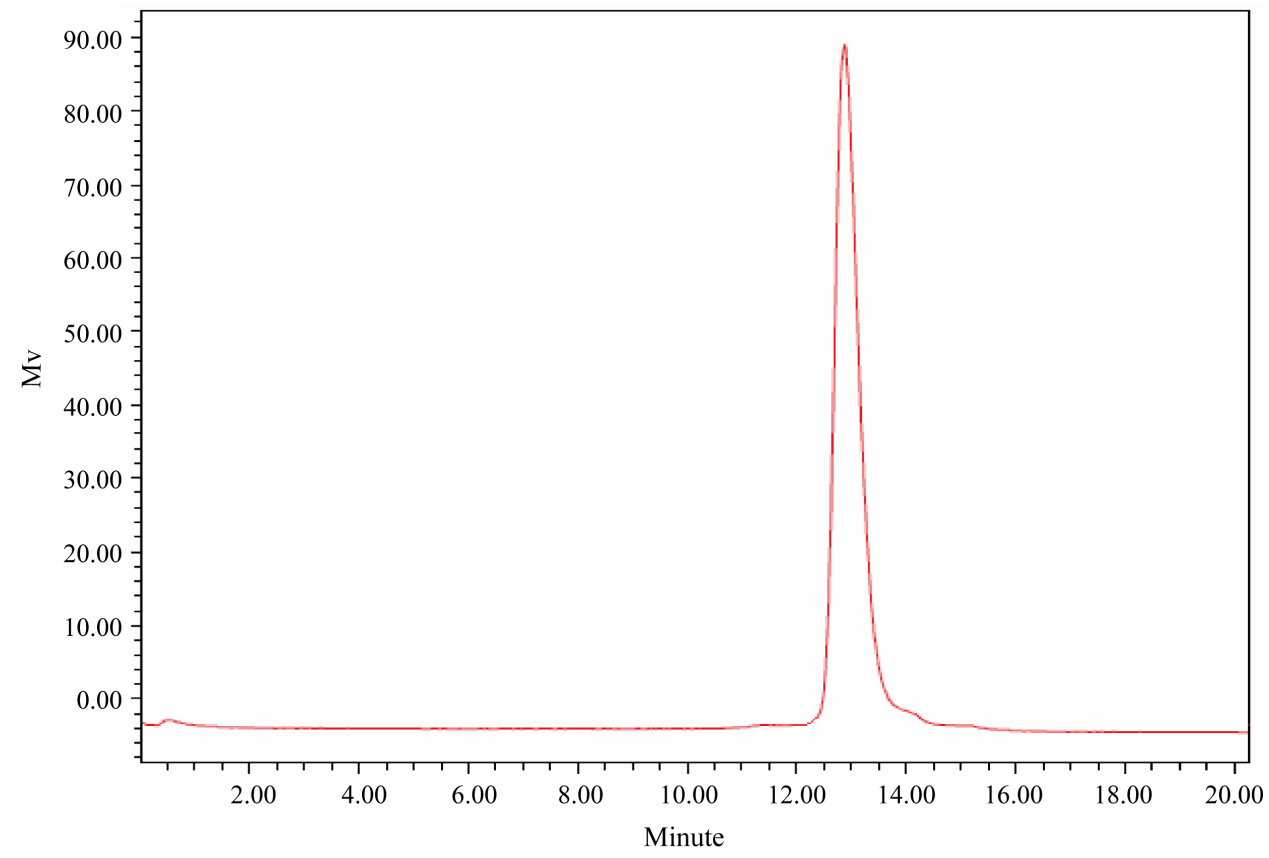

(b)

Figure 4. (a) HPLC-ELSD chromatogram of ESP-A1 with sugar KS-805; (b) HPLC-ELSD chromatogram of ESP-A2 with sugar KS-805.

\subsection{Monosaccharide Composition of Polysac- charide and its Molar Ratio}

The lack of chromophores or fluorophores in the structure of monosaccharides limits their sensitive detection in HPCE $[7,8]$. Therefore, carbohydrates are generally tagged with a suitable chromophore or fluorophore to obtain highly sensitive detection. The reagent 1-phenyl-3 -methyl-5-pyrazolone (PMP) is one of the popular labels that react with reducing carbohydrate under mild condition, requiring no acid catalyst and causing no isomerization [6-8]. This experiment was designed to develop a 

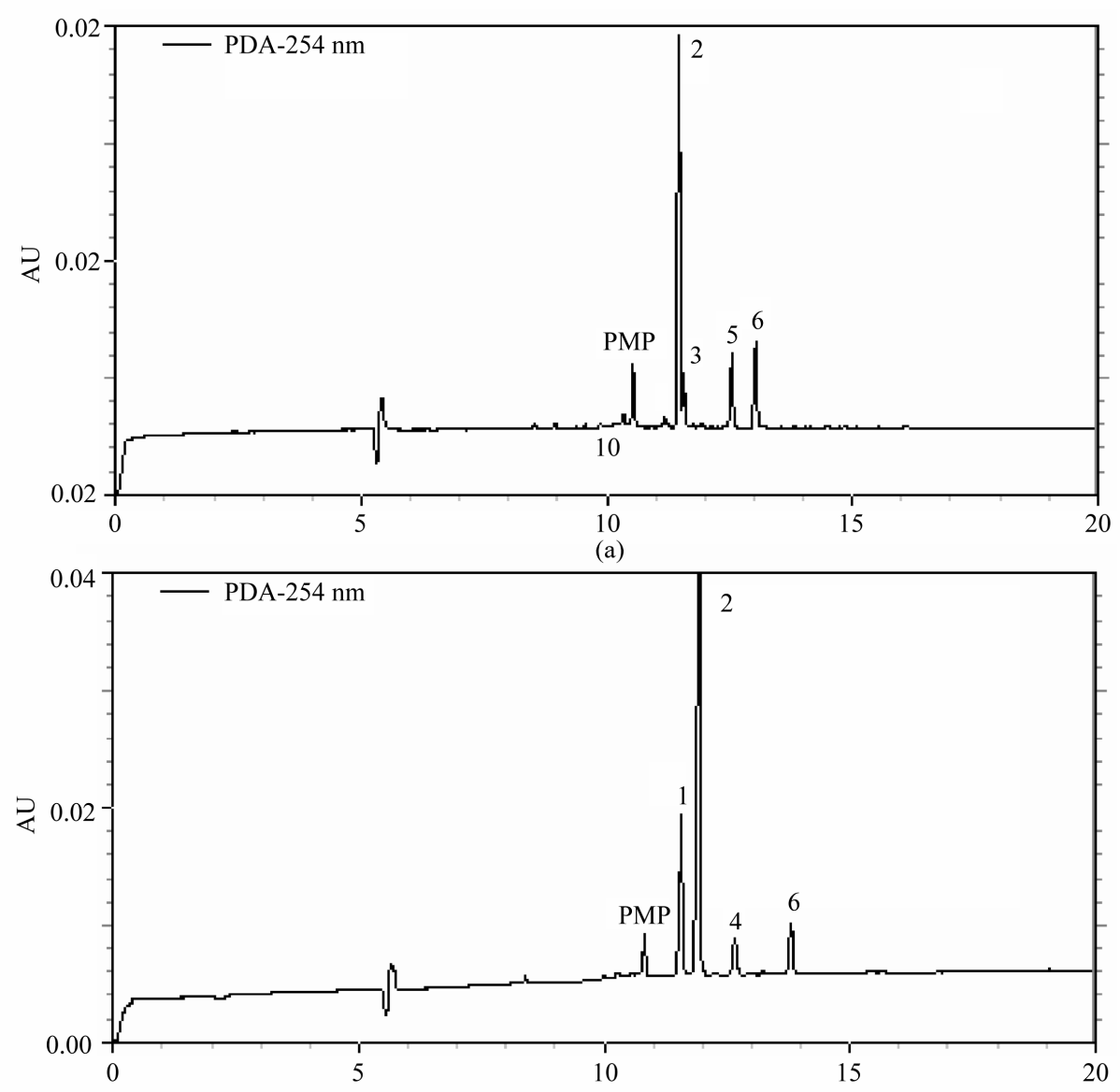

(b)

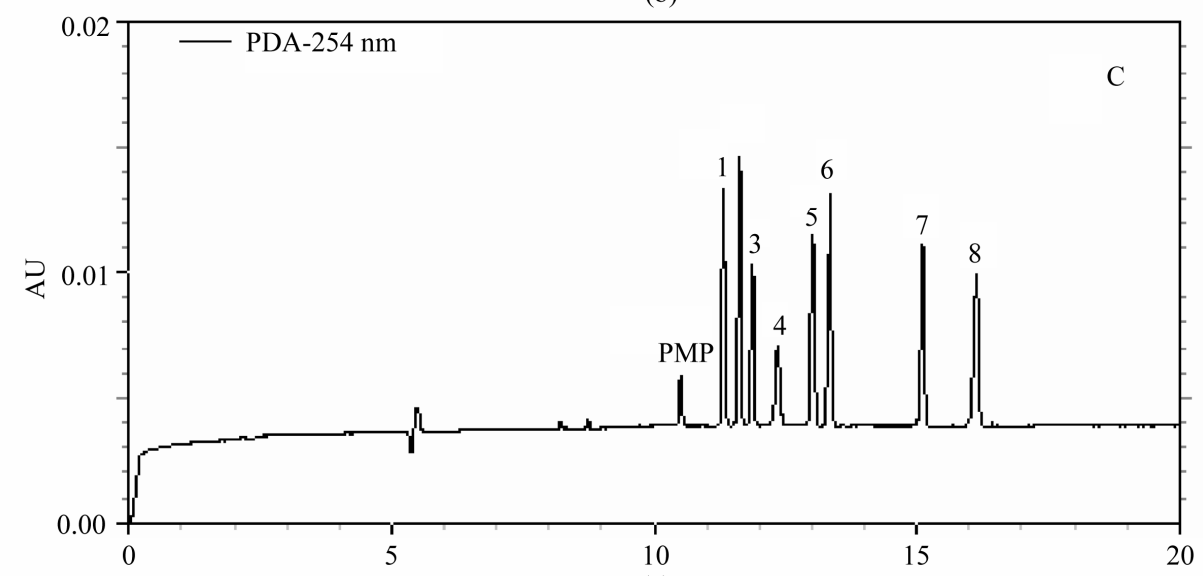

(c)

Figure 5. Electropherograms of PMP derivatives of monosaccharides in ESP-A1 (a), ESP-A2 (b) and standard monosaccharides (c). Separation condition: $35 \mathrm{mM}$ borate at $\mathrm{pH} 10.02$, capillary temperature $25^{\circ} \mathrm{C}$ and applied voltage 20 kV, 0.2 mM each. Peak identities: 1, Xyl; 2, Ara; 3 Glc; 4 Rha; 5, Man; 6, Gal; 7, GlcUA; 8, GalUA. Detection, $254 \mathrm{~nm}$ direct mode; injection pressure, 0.5 psi for $5 \mathrm{~s}$; capillary, fused-silica $48.5 / 58.5 \mathrm{~cm}$ (Ldet/Ltot); separation temperature, $25^{\circ} \mathrm{C}$.

rapid, repeatable and accurate CZE method with PMP pre-column derivatization for the quantification of the component carbohydrates in the cold water-soluble polysaccharides from the stems of E. sinica. In the proc- ess of structural analysis of polysaccharides, the molar ratio of each monosaccharide in polysaccharides is one of the most important parameters. It is unnecessary to obtain the specific amount of each monosaccharide be- 
cause researchers just want to know the molar ratios of each one. Therefore, the molar ratio can be calculated by the equation $\left[R_{i / n}=f_{i / n} \times\left(A_{i}^{\prime} / A_{n}^{\prime}\right)\right]$ rather than specific amount of each sugar obtained. We believe that the former is more favorable in the elimination of systematic errors.

We have studied hydrolysis conditions of ephedra polysaccharides and have found that $6 \mathrm{~h}$ at $110^{\circ} \mathrm{C}$ with $2 \mathrm{M} \mathrm{H}_{2} \mathrm{SO}_{4}$ gave nearly quantitative release of neutral and acidic sugars from several polysaccharides [6]. Therefore, our previous method was used in this work. ESP-A1 and ESP-A2 were hydrolyzed with $2 \mathrm{M} \mathrm{H}_{2} \mathrm{SO}_{4}$, and PMPlabeled as described in the experimental section and finally, the released monosaccharide derivatives were analyzed by the described CZE method under the optimized conditions. Figures 5(a) and 5(b) showed typical chromatograms of the cold water-soluble two polysaccharide samples. As can be seen, the PMP derivatives of the component monosaccharides released from the ESP-A1 and ESP-A2 could be still baseline separated and the component monosaccharides could be identified by comparing with the chromatogram of the mixture of standard monosaccharides (Figure 5(c)). Each monosaccharide peak in the order of increasing retention time was identified as xylose, arabinose, glucose, rhamnose, mannose, galactose, glucuronic acid and galacturonic acid. It was shown that ESP-A1 consisted of xylose, arabinose, glucose, mannose and galactose and ESP-A2 consisted of xylose, arabinose, rhamnose and galactose, in a molar ratio (mol\%) of $3.2: 61.1: 11.1: 12.9: 11.6$ and 20.6: 67.7: 5.0: 6.7 , respectively. It was clear that the predominantly composition monosaccharide in ESP-A1 and ESP-A2 was arabinose up to $61.1 \%$ and $67.7 \%$ $(\mathrm{mol} \%)$, respectively.

\section{Conclusion}

Two polysaccharides extracted from the stems of $E$. sinica (ESP-A1 and ESP-A2) were successfully separated and purified. The results reported in this paper provided an excellent example for the isolation, purification, determination of molecular weights and molar ratios among monosaccharides of complex hetero-lysaccharide from E. sinica. The present finding provides a basis for further structural analysis and evaluation of the bioactivities of $E$. sinica polysaccharides for its application in food and medicinal fields.

\section{Acknowledgements}

Our work was supported by the Major State Basic Research Development Program of China (973 Program 2006CB504708), State Key Creative New Drug Project of 12th Five-year Plan of China (2011ZX11102), National Natural Science Foundation of China (No. 30973870) and Heilongjiang University of Chinese Medicine Doctor Innovative Foundation.

\section{References}

[1] Jiangsu New Medical College, "Dictionary of Chinese Materia Medica," Shanghai People's Press, Shanghai, 1986

[2] H. X. Kuang, Y. G. Xia and B. Y. Yang, et al., "Screening and Comparison of the Immunosuppressive Activities of Polysaccharides from the Stems of Ephedra sinica Stapf," Carbohydrate Polymers, Vol. 83, No. 2, 2011, pp. 787-795.

[3] Y. G. Xia, H. X. Kuang and B. Y. Yang, et al., "Optimum Extraction of Acidic Polysaccharides from the Stems of Ephedra sinica Stapf by Box-Behnken Statistical Design and Its Anti-Complement Activity," Carbohydrate Polymers, doi:10.1016/j.carbpol.2010.11.035.

[4] W. A. Trujillo and W. R. Sorenson, "Determination of Ephedrine Alkaloids in Human Urine and Plasma by Liquid Chromatography/Tandem Mass Spectrometry: Collaborative Study," Journal of AOAC International, Vol. 86, No. 4, 2003, pp. 643-656.

[5] A. M. Staub, "Removeal of Protein-Sevag Method," Methods in Carbohydrate Chemistry, Vol. 5, 1965, pp. 5-6.

[6] Y. G. Xia, Q. H. Wang, J. Liang, et al., "Development and Application of A Rapid and Efficient CZE Method Coupled with Correction Factors for Determination of Monosaccharide Composition of Acidic Hetero-Polysaccharides from Ephedra sinica," Phytochemical Analysis, doi:10.1002/pca.1235.

[7] F. Daotian and A. Roger, "Monosaccharide Composition Analysis of Oligosaccharides and Glycoproteins by HighPerformance Liquid Chromatography," Analytical Biochemistry," Vol. 227, No. 2, 1995, pp. 377-384.

[8] Y. Lv, X. B. Yang, Y. Zhao, et al., "Separation and Quantification of Component Monosaccharides of the Tea Polysaccharides from Gynostemma pentaphyllum by HPLC with Indirect UV Detection," Food Chemestry, Vol. 112, No. 3, 2009, pp.742-746. 